Research paper

\title{
Development of a novel mechanical micro-engraving method for the high-aspect-ratio microstructures of an advanced window system
}

\author{
Jing Gong ${ }^{\mathrm{a}, *}$, Antoine Delaunay ${ }^{\mathrm{a}}$, André Kostro ${ }^{\mathrm{b}}$, Andreas Schüler ${ }^{\mathrm{a}}$ \\ a Solar Energy and Building Physics Laboratory, Ecole Polytechnique Fédérale de Lausanne (EPFL), CH-1015 Lausanne, Switzerland \\ b BASF Schweiz AG, Klybeckstrasse 141, CH-4057 Basel, Switzerland
}

\section{A R T I C L E I N F O}

\section{Article history:}

Received 7 October 2017

Received in revised form 28 January 2018

Accepted 30 January 2018

Available online 1 February 2018

\section{Keywords:}

Advanced glazing

Scanning mechanical engraving

Large-area microfabrication

Curve side-wall microstructures

High aspect-ratio microstructures

\begin{abstract}
A B S T R A C T
A transparent polymer film with embedded micro mirrors is developed, and it can be attached to a window glass pane for daylighting, improving visual comfort and reducing cooling load in buildings by redirecting sunlight. Despite existing cutting-edge techniques, the fabrication of large-area, linear micro structured surfaces combining high aspect ratio, curve sidewalls and a controlled shape remains challenging. In order to solve the problem, a novel mechanical scanning engraving method using blades with micro-teeth of desired patterns on soft substrates is developed. The usage of a blade enables the combination of high aspect ratio with almost any closed profile. Such blades can be obtained in a large variety of shapes using UV-LIGA technology which combines photolithography and electroforming. Master molds were prepared and the following steps of micro UV-imprinting from the master molds are also introduced. The present work confirms the feasibility of the fabrication for parabolic sidewall microstructures of aspect ratio of 3.8 and 6.7 with good controlled shapes. With a scanning speed of $0.15 \mathrm{~m} / \mathrm{min}$, the method is proven to be efficient and appropriate for large-area microstructured fabrication.
\end{abstract}

(C) 2018 Elsevier B.V. All rights reserved.

\section{Introduction}

It is stated that the energy consumption by both residential and commercial buildings in developed countries account for $20-40 \%$ of total energy used [1]. This energy is mainly used for space heating, cooling and lighting. Windows of building envelope can cause severe overheating in summer and large thermal loss in winter due to its relatively high solar gain factor and high conductance, respectively. However, a window allows the penetration of natural light into buildings and builds the connection between occupants and exterior environment. The biological benefits of natural lightings and exterior view has been confirmed by researches. Therefore, fenestration design needs to keep a balance energy performance of building while fulfil its nature of providing daylight and external view. In order to achieve the appropriate fenestration design, we are developing a multifunctional window system based on embedded micro-mirrors in a thin polymer layer [2,3]. The thin polymer layer can be laminated with a glass pane, and the basic principle of the system is shown in Fig. 1. A polymer layer consists of a periodic array of parabolic reflective surfaces for light-redirecting coupled with an array of secondary reflective surfaces for lightblocking. In winter (Fig.1(a)), the incoming solar radiation is redirected by the reflective parabolic surface to reach the rear of a room. In contrast, in summer (Fig. 1(b)) where the elevation angle is high, the direct

\footnotetext{
* Corresponding author.

E-mail address: jing.gong@epfl.ch (J. Gong).
}

solar radiation is focused on secondary reflective surfaces and reflected outside. Through this method, seasonal thermal control is achieved. Due to the redirecting mirrors, up to $80 \%$ of direct solar light can be redirected toward the ceiling to extend the area of daylight autonomy [3]. Thanks to the redirection of the daylight, glare is reduced and the visual comfort can be improved in an interior space [3]. It should be noted that, the projected height of the mirrors on the vertical direction is in the order of $10 \mu \mathrm{m}-50 \mu \mathrm{m}$; moreover, the first surface for incoming rays and the end surface for out-going rays are flat and parallel. Thus a clear view through the glazing with embedded micro-mirrors may be achieved.

The window system with micro-mirrors requires the fabrication of a master mold with well-defined microstructures of aspect ratio larger than 3 on a window-sized area. One or several steps of replications to translate the pattern from the mold to a polymer film attached to a glass substrate are then needed. However, concerning the master mold, the fabrication of microstructures with aspect ratio larger than 3 for window-sized area remains challenging. Electrical discharge machining (EDM) makes use of electrical energy and turns it into thermal energy through a series of discrete electrical discharges occurring between the electrode and workpiece immersed in a dielectric fluid [4]. By nature EDM is made for cutting pieces but not to fabricate a large area microstructured surface for the use of windows. Lift-off lithography refers to the process of exposing a pattern into photoresist, depositting a thin film over the entire area, and then washing away the photoresist to leave behind the film only in the patterned area. However, lift-off lithography is difficult to fabricate curve sidewalls with controlled optical 
(a)

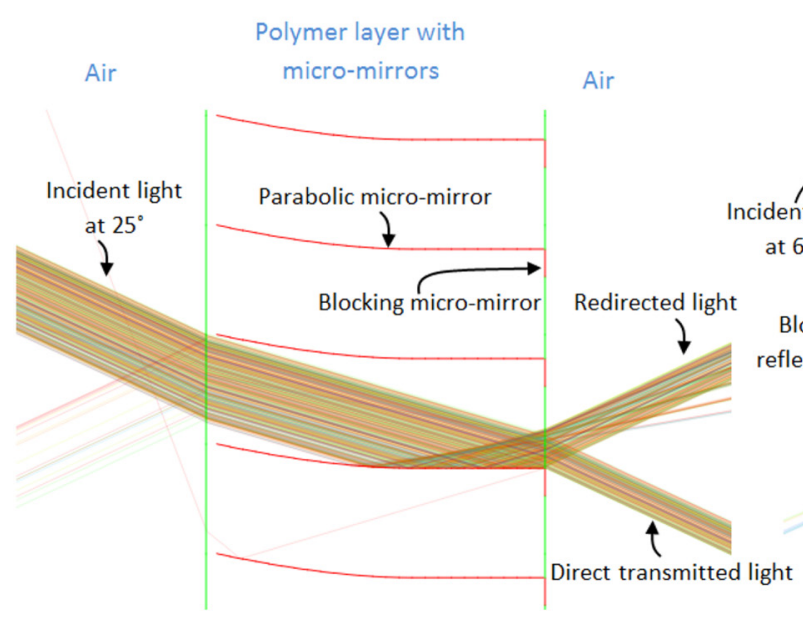

(b) Summer

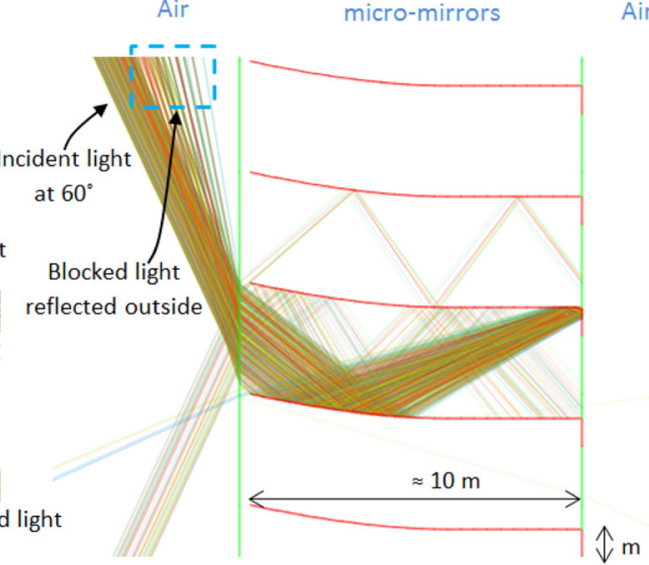

Fig. 1. (a) Modeling of embedded micro-mirrors in a polymer layer for ray-tracing simulation to optimize the geometry of the micro-mirrors: (a) in winter and (b) in summer.

surface [5]. Gray-scale direct laser writing uses masks with changing opacity makes it possible to fabricate curved side wall microstructures [6]. However, the technique requires high-end equipment and the procedures are delicate. Moreover the microstructured area is constrained by the size of the mask. Patterns obtained by interference lithography or scanning UV laser ablation uses a complex mask pattern to project onto the surface of the workpiece which is moved at constant velocity to form a linear structure of constant profile or accelerated or decelerated to form curve structures [7]. However, due to the complex physical and or chemical interaction between debris and laser, for the microstructures of aspect-ratio larger than 3 , it can be difficult to have controlled shapes. Mechanical scanning engraving methods with ultraprecision single point diamond, such as cutting fly-cutting [8], nonrotational cutting $[9,10]$ and raster milling [11] have been developed.
Micro-grinding with the truing of diamond wheel [12] has been used for arrays of micro $V$ grooves. However, these mechanical scanning engraving methods are single-line scanning method; a high-precision displacement sensor and delicate control system are needed to guarantee the accurate alignment between two arrays of grooves.

In the present work, a novel mechanical multi-line scanning method for large area fabrication of linear microstructures is developed. The following procedures of micro UV-imprinting from the master mold steps are also introduced. The feasibility for the fabrication of Parabolic-sidewall microstructures of aspect ratio of 3.8 and 6.7 are confirmed.

The method is initially developed for the glazing with micro-mirrors. However, it can also be applied to rapid prototyping of micro lens arrays (e.g. micro $\mathrm{V}$ grooves) for grating [13], retro-reflection [14] and other light-guiding [15]. Furthermore, it can be also used for the fast
Scanning engraving
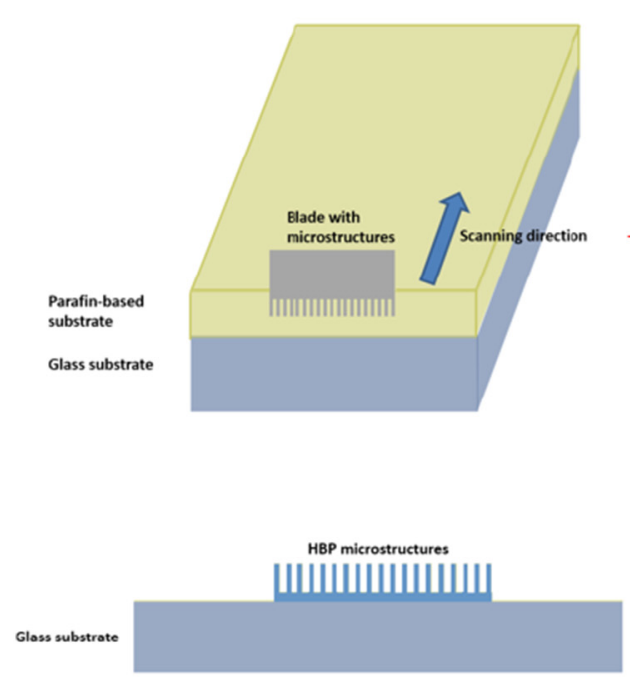

\section{UV curing}

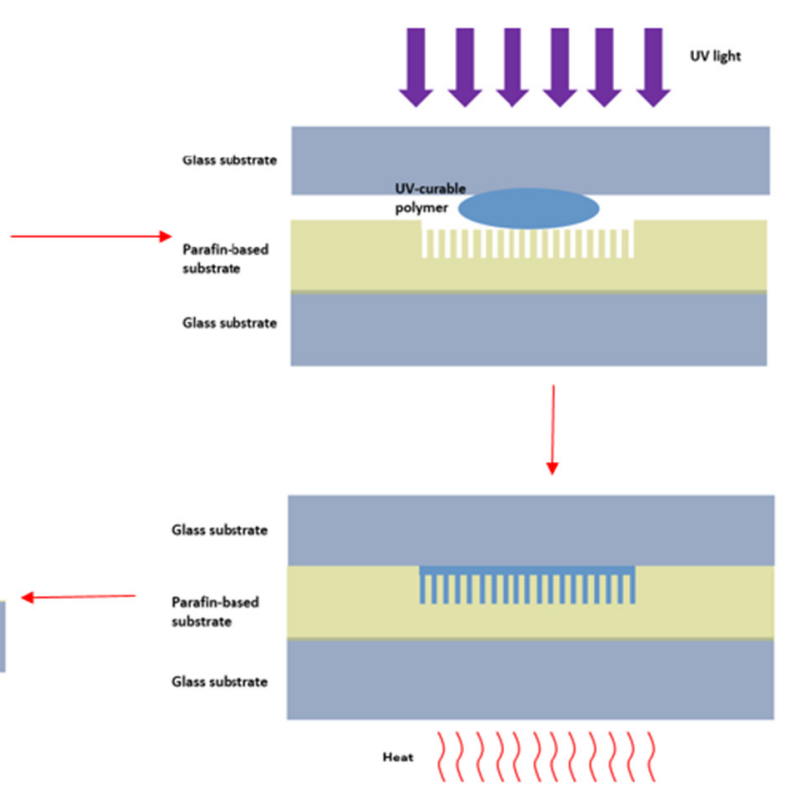



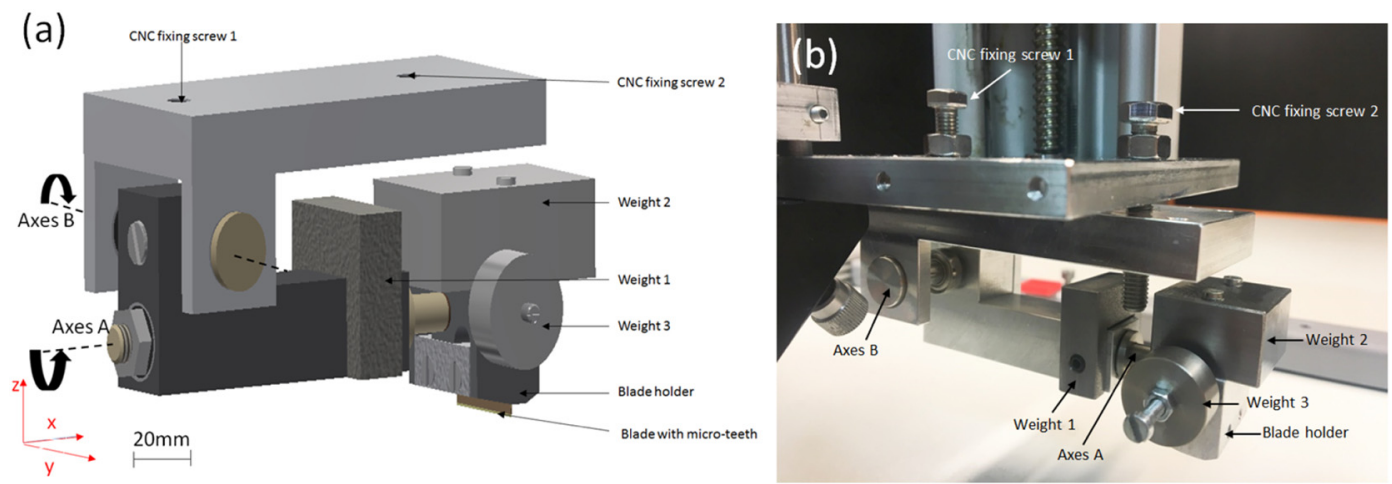

Fig. 3. (a) A schematic design of the head which is installed on a CNC machine with the blade. (b) The fabricated head based on the design.

fabrication of artificial superhydrophobic surfaces with patterned microstructures [12,16,17]. Additionally, it has good potential of the realization of microfluidic channels for cell biology research [18] and protein crystallization [19].

\section{Designs and preparation}

Prior to the fabrication of the optical microstructures, rigorous optimization of the geometry for the curvature, the alignment and the dimensions of the micro mirrors is conducted by ray-tracing simulation using the software CFSpro [20]. Ray-tracing simulation is done with calculating the path of waves or particles through a system with regions of varying propagation velocity, absorption characteristics, and reflecting surfaces. It is found that, the effective ratio of the dimension between redirecting mirrors and the blocking mirrors normally ranges from 3 to 10 . The fabrication process for the optical microstructures was divided into five steps: substrate preparation, engraving, UV imprinting, mold removing and cleaning. The details of the five steps are described as follows: (1) Firstly, paraffin substrates mixed with bee wax are prepared. (2) Secondly, the micro-toothed blade was installed on a special designed head attached to a CNC machine, and the substrate is fixed on a stage. Then the blade was dragged through the soft material in a direction perpendicular to the profile with enough pressure. The aim consisted in obtaining a negative of the comb profile in the soft material. (3) The obtained mold is then printed onto a UV-curable liquid acrylated hyper-branched polymer (HBP) and exposed to UV light.

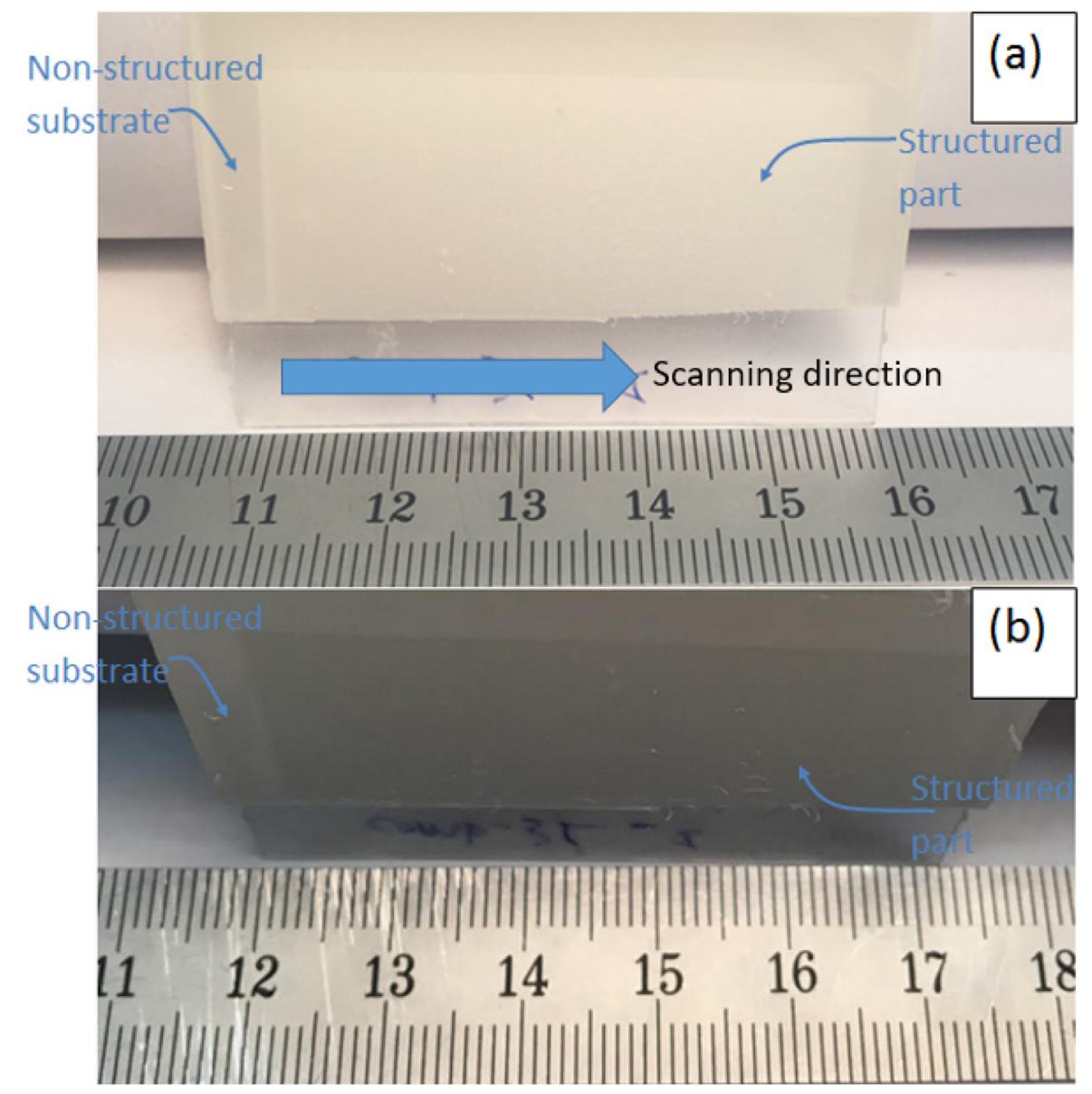

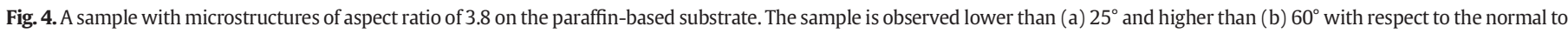
the surface. 
(4) Once the polymer is cured by the UV light, the master mold is molten on a heat plane with a temperature of about $60^{\circ} \mathrm{C}$. (5) Finally the obtained HBP microstructures with the opposite pattern with respect to the paraffin-based master mold are immersed in universal solvent (DISSOLV. CELLUL. 201, Brenntag) and cleaned in ultra-sonic bath for about 10 min. Fig. 2 presents the sketch of the process flow from master mold fabrication to HBP microstructures on a glass substrate.

The method is based on engraving a soft material using a blade with patterned micro-teeth. Paraffin-based substrates with $10 \mathrm{wt} \%$ beeswax are used in the present study. The optimized patterns of the microteeth are shown in Fig. 5(a) and (b). The shape of microstructures is either the positive or the negative counterpart of the microstructures on a glass substrate. The functional parts of the microstructures consists of a controlled parabolic surface for the preparation of the redirecting mirror and a platform for the blocking mirror. The aspect ratio is defined as the height of the structure over the width at mid-height due to the trapezoidal shapes of the microstructures [21], and they are 3.8 and 6.7, respectively. The blade with patterned micro-teeth is a $150-\mu \mathrm{m}$-thick in nickel, and it is fabricated with the UV-LIGA technique which combines photolithography and electroforming [22] by Mimotec SA. The roughness of the surface on the nickel master mold does not exceed $0.2 \mu \mathrm{m}$.

A head on which the blade is fixed and attached was designed to install on a Computer Numeric Control (CNC) machine with a positioning resolution of $10 \mu \mathrm{m}$. The design of the head and the defined coordinates are shown in Fig. 3. Screw 1 and screw 2 are used to install the head to a $\mathrm{CNC}$ machine. Two axes are used in the present work. Axes A allows the rotation around $X$ axis and ensure the placement of the blade is parallel to the substrate. Axes $B$ enables the rotation around the $Y$ axis and allows the linear movement of the sample holder along the y direction. Screw 1 will fix the sample holder at the certain position but the rotation around y axis is possible. During engraving, the force applied on the blade $\mathrm{A}$ is equivalent to about $7.8 \mathrm{~N}$ and the force on the blade $\mathrm{B}$ is approximately $9.8 \mathrm{~N}$. The scanning engraving is conducted at room temperature in ambient condition.
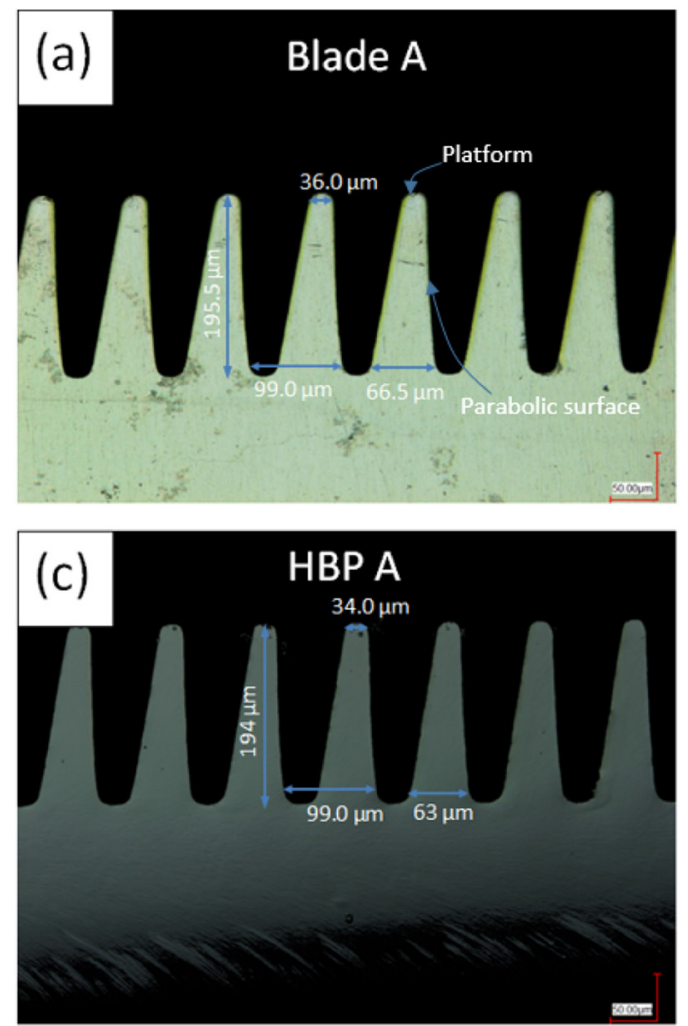

After engraving, the mold is placed on a flat plane. Then liquid crylated HBP is dropped and a glass substrate is put on the mold. After the liquid HBP fully fill the microstructured area, an exposure to UV light at an intensity of around $8 \mathrm{~mW} / \mathrm{cm}^{2}$ with the emitted wavelength of $375 \mathrm{~nm}$ for $180 \mathrm{~s}$ is conducted. The UV curing is carried out at room temperature in ambient condition. After that, the sample was flipped and exposed to the same intensity for another $180 \mathrm{~s}$, in order to ensure homogeneous photoconversion of the $300 \pm 20 \mu \mathrm{m}$ thick sample. Once the polymer is cured by the UV light, the mold is melted on a heat plate (brand name) with a temperature of about $60{ }^{\circ} \mathrm{C}$ in ambient condition. Finally the obtained HBP microstructures with the opposite pattern with respect to the paraffin-based master mold are immersed in universal solvent (DISSOLV. CELLUL. 201, Brenntag) and cleaned in an ultra-sonic bath for about 10 min. Then the HBP sample was further immersed in ethanol in the ultrasonic bath for approximately 5-15 min, and naturally dried in air.

\section{Results}

Fig. 4 shows a sample with microstructures of aspect ratio of 3.8 on the paraffin-based substrate. The sample is observed lower than $25^{\circ}$ (Fig. 5(a)) and above $60^{\circ}$ (Fig. 5(b)) in diffused daylight. Light of incident angle lower $25^{\circ}$ is partially redirected to eyes. Therefore the microstructured part appears brighter than the non-structured part. In contrast, light of incident angle higher than $60^{\circ}$ is redirected to the blocking surface and then reflected back to the coming side. Thus the microstructured part looks darker than the non-structured part. The redirection effect and the blocking effect due to the optimized geometry are preliminarily confirmed. The microstructured area is about $45 \mathrm{~mm}$ $\times 25 \mathrm{~mm}$, corresponding to one linear scan of the 25 -mm wide blade along the substrate for $45 \mathrm{~mm}$. The speed of the scanning is $150 \mathrm{~mm} / \mathrm{min}$. Hence, a $45-\mathrm{mm}$ scan takes $20 \mathrm{~s}$.

Fig. 5 shows the cross-sections of the blade A and B and their corresponding HBP microstructures. For the blade $A$, the average depth of the micro teeth is $195.5 \pm 0.5 \mu \mathrm{m}$, and the width of the two ends of a teeth are $36 \pm 0.3 \mu \mathrm{m}$ and $66.5 \pm 0.5 \mu \mathrm{m}$, respectively. The corresponding
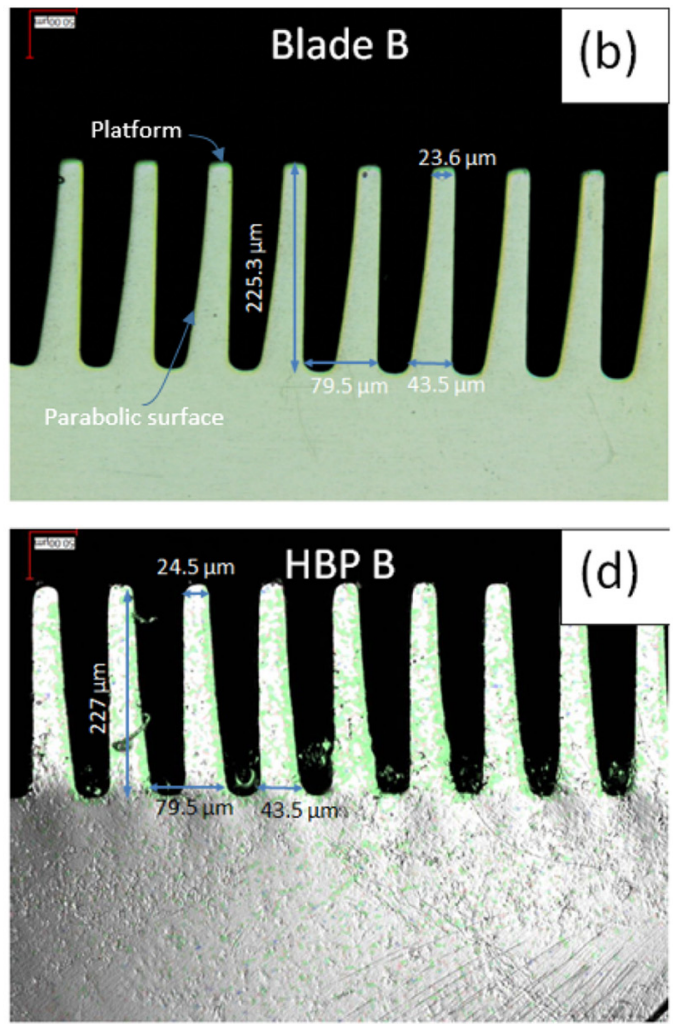

Fig. 5. The cross-sections of the blade A and B and their corresponding HBP microstructures. 
dimensions of the polymer microstructures HBP A are $194 \pm 0.5 \mu \mathrm{m}, 34$ $\pm 0.3 \mu \mathrm{m}$ and $66.5 \mu \mathrm{m}$. It is likely due to the shrinkage of polymerization [23], the dimensions of microstructures HBP A are slightly reduced. Nonetheless, the relative reduction is below $5 \%$. For the blade $\mathrm{B}$, the average depth of the micro teeth is $225.3 \pm 0.5 \mu \mathrm{m}$, and the width of the two ends are $23.6 \pm 0.3 \mu \mathrm{m}$ and $43.5 \pm 0.5 \mu \mathrm{m}$, respectively. Correspondingly, the dimensions of the polymer microstructure HBP B are $227 \pm 3 \mu \mathrm{m}, 24.5 \pm 0.5 \mu \mathrm{m}$ and $43.5 \pm 0.5 \mu \mathrm{m}$, respectively. It is shown that the dimensions of the polymer microstructures HBP B are slightly increased by maximum $3.8 \%$ can be due to the swelling $[24,25]$ of polymer during cleaning in the dissolve solvent. Nonetheless, the swelling can be suppressed by the reduction of cleaning time with the improvement of the cleaning procedures. Overall, the observation of the cross-sections shows the good control of shape by the method.

In order to study the shape fidelity, for both the blades and the polymer microstructures, the morphology of the parabolic surfaces (examples marked in Fig. 5(a) and (b)) and the platforms of the microstructures are extracted for ray-tracing simulation using the software CFSpro [19]. The reflectance of the extracted surfaces are set as 0.9 to serve as mirrors. The alignment between the parabolic mirrors and the blocking mirrors is identical to the example shown in Fig. 1. In the computer model, the polymer layer is laminated to the inner surface of the outer glass pane of a double glazing. A reference case is taken with the conventional double glazing. The angular dependent transmittance is calculated. Fig. 6 shows the simulated angular-dependent transmittance: transmittance in the visible range for different incident angles at the azimuth of $0^{\circ}$. For the case $A$ and case $B$, there is a decrease of the transmittance from $0^{\circ}$ to $20^{\circ}$. It is mainly because that in this angular range, a fraction of incoming light directly goes to the blocking mirror without being redirected by the parabolic mirrors and then is reflected to exterior. The application of such angular feature can be used in the place where the winter is mild or the elevation angle of the winter sun for the working hours are higher than $20^{\circ}$. For the blade $A$ and the polymer microstructure HBP A, the simulated transmittances are highly similar, confirming the good shape preservation and surfaces with similar roughness. The profiles of the angular-dependent transmittances are identical to the case of the conventional double glazing in the angular range from about $20^{\circ}$ to $55^{\circ}$, and then the transmittance moderately reduce from $55^{\circ}$ to $75^{\circ}$. Such designs would be used mainly for light redirection and glare protection, as they do not offer a strong angular dependence. For blade B and the polymer microstructure HBP B, the trends of the profiles are similar but the deviation is observed. The transmittance drop is shifted by about $7^{\circ}$ toward the normal incidence. The shift is likely due to two reasons: designs with higher aspect ratio are more sensitive to variation in the curvature of the mirrors and the variation in shape during engraving and replication also tends to increase. Nonetheless, a strong angular dependence of the transmittance is achieved. When the target is to moderate solar gains in summer but maximize them in winter, such a design is desired.

Compared with blade $A$, the strong angular dependence of blade $B$ is due to its higher aspect ratio of the microstructures and the higher curvature of the parabolic surface. More direct light will be redirected for micro mirrors of higher aspect-ratio micro-mirrors. Higher curvature of the parabolic surface make light of certain incident angles more effectively focused onto the blocking mirror. Therefore, blade B results in the system of embedded micro-mirrors with significantly decreased transmittance at high incident angles, compared to blade A.

\section{Conclusions and outlook}

The present work focuses on master mold fabrication and microstructure transferred on a glass substrate. A novel mechanical engraving method using blades with micro-teeth of desired patterns for the preparation of master molds is developed. The following steps of UVmicroimprinting from the master molds are also developed. The fabrication for parabolic-side-wall microstructures of aspect ratio of 3.8 and 6.7 with good controlled shapes is achieved. The desired angulardependent optical properties are obtained. With a scanning speed of $15 \mathrm{~cm} / \mathrm{min}$, the method has the potential to be efficient and appropriate for large-area microstructured fabrication. In the future, the relation between the added weights (pressure) on blades and engraved depths will be investigated in the future. The different ratio between paraffin and beeswax, which is related to the hardness and the brittleness of the substrate, should also be investigated. Appropriate alternatives for the engraved substrate will be considered. The influence of the scanning speed will be studied. For further scaling up to window-size prototype, effective ways to remove the debris during scanning will be developed. Proper programming in the CNC machine for stitching is also necessary. More structural characterization will be conducted, and the possible oscillation will be identified. Accordingly, upgrading of the tools for damping the possible oscillation will also be carried out. Regarding the following experiments and characterization of samples, deposition of micro-mirrors with highly reflective materials will be conducted on the HBP microstructures and encapsulation will be done. After achieving samples with encapsulated micro-mirrors, experimental optical measurement with goniophotometer will be performed.
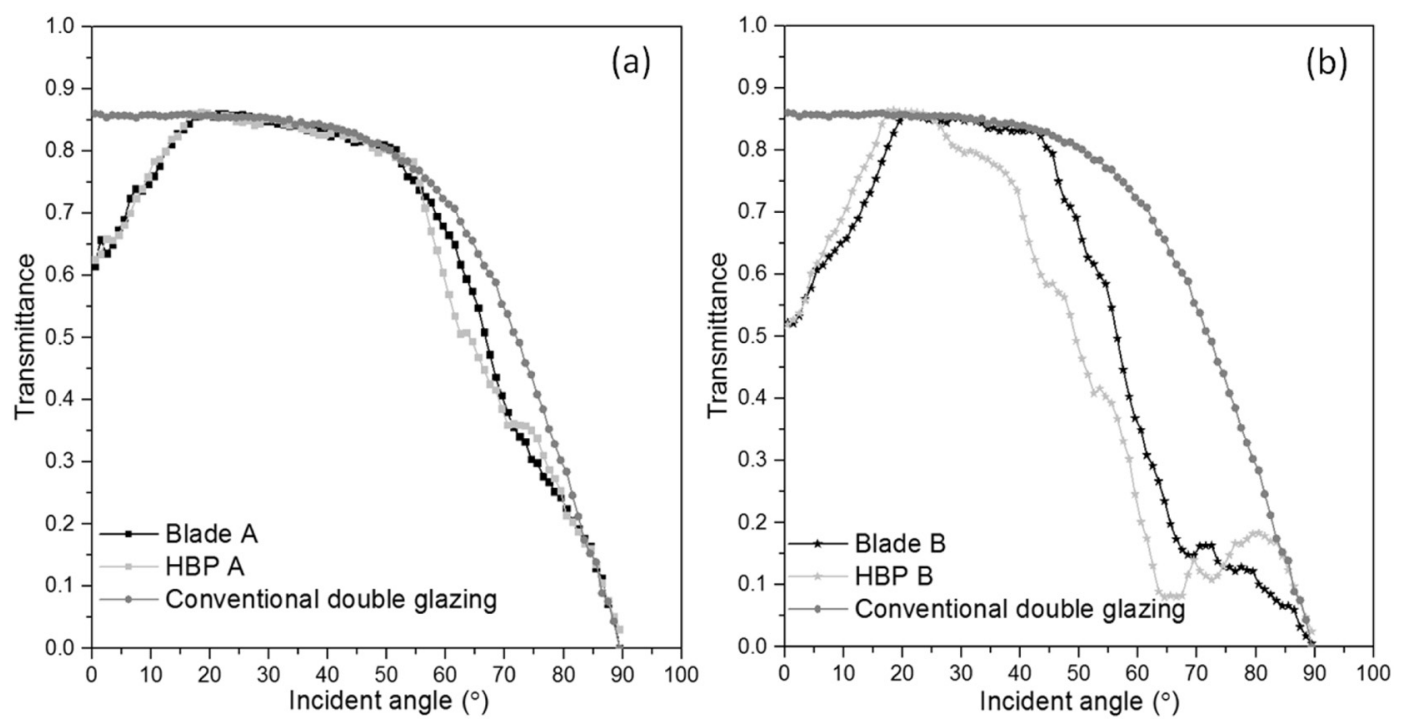

Fig. 6. The simulated angular-dependent transmittance for blade A and B and their corresponding HBP microstructures. 


\section{Acknowledgement}

We would like to thank the Swiss Competence Center for Energy Research (SCCER) (grant NO. REF-1115-40004) for funding of this project.

\section{References}

[1] L. Pérez-Lombard, J. Ortiz, C. Pout, A review on buildings energy consumption information, Energ. Buildings 40 (3) (2008) 394-398.

[2] A. Kostro, M.A. Gonzalez Lazo, Y. Leterrier, E. Siringil, P. Hoffmann, A.M. Schüler, Laser ablation and nanoimprint lithography for the fabrication of embedded light redirecting micromirrors, Proceedings of International Conference CISBAT $2015 \mathrm{Fu}$ ture Buildings and Districts Sustainability from Nano to Urban Scale (No. EPFLCONF-213287, pp. 15-20), LESO-PB, EPFL, 2015.

[3] J. Gong, A. Kostro, A. Motamed, A. Schueler, Potential advantages of a multifunctional complex fenestration system with embedded micro-mirrors in daylighting, Sol. Energy 139 (2016) 412-425.

[4] K.H. Ho, S.T. Newman, State of the art electrical discharge machining (EDM), Int. J. Mach. Tools Manuf. 43 (13) (2003) 1287-1300.

[5] D. Bratton, D. Yang, J. Dai, C.K. Ober, Recent progress in high resolution lithography, Polym. Adv. Technol. 17 (2) (2006) 94-103.

[6] C.H. Tien, Y.E. Chien, Y. Chiu, H.P.D. Shieh, Microlens array fabricated by excimer laser micromachining with gray-tone photolithography, Jpn. J. Appl. Phys. 42 (3R) (2003) 1280

[7] H. Hocheng, K.Y. Wang, Microgroove pattern machined by excimer laser dragging Int. J. Manuf. Technol. Manag. 13 (2-4) (2008) 241-253.

[8] F.Z. Fang, Y.C. Liu, On minimum exit-burr in micro cutting, J. Micromech. Microeng. 14 (7) (2004) 984.

[9] T. Moriya, K. Nakamoto, T. Ishida, Y. Takeuchi, Creation of V-shaped microgrooves with flat-ends by 6-axis control ultraprecision machining, CIRP Ann. Manuf. Technol. 59 (1) (2010) 61-66.

[10] Y. Takeuchi, M. Murota, T. Kawai, K. Sawada, Creation of flat-end V-shaped microgrooves by non-rotational cutting tools, CIRP Ann. Manuf. Technol. 52 (1) (2003) 41-44.

[11] S. Wang, To, S, X. Chen, H. Wang, H. Xia, A study of the fabrication of v-groove struc ture in ultra-precision milling, Int. J. Comput. Integr. Manuf. 27 (11) (2014) 986-996.
[12] P. Li, J. Xie, J. Cheng K.K. Wu, Anisotropic wetting properties on a precision-ground micro-V-grooved Si surface related to their micro-characterized variables, J. Micromech. Microeng. 24 (7) (2014), 075004. .

[13] H. Ohmori, N. Ebizuka, S. Morita, Y. Yamagata, H. Kudo, Ultraprecision microgrinding of germanium immersion grating element for mid-infrared super dispersion spectrograph, CIRP Ann. Manuf. Technol. 50 (1) (2001) 221-224.

[14] C.Y. Lin, C.H. Su, C.M. Hsu, C.R. Lin, Improvement of the microcrystalline cube corner reflective structure and efficiency, Jpn. J. Appl. Phys. 47 (7R) (2008) 5693.

[15] A. Priyadarshi, L.H. Fen, S.G. Mhaisalkar, V. Kripesh, A.K. Asundi, Fiber misalignment in silicon V-groove based optical modules, Opt. Fiber Technol. 12 (2) (2006) 170-184.

[16] F. Varnik, M. Gross, N. Moradi, G. Zikos, P. Uhlmann, P. Müller-Buschbaum, ... M. Stamm, Stability and dynamics of droplets on patterned substrates: insights from experiments and lattice Boltzmann simulations, J. Phys. Condens. Matter 23 (18) (2011) 184112.

[17] L.B. Kong, C.F. Cheung, To, S, C.T. Cheng, Modeling and characterization of generation of 3D micro-structured surfaces with self-cleaning and optical functions, Optik 124 (17) (2013) 2848-2853.

[18] S.M. Kim, S.H. Lee, K.Y. Suh, Cell research with physically modified microfluidic channels: a review, Lab Chip 8 (7) (2008) 1015-1023.

[19] N.E. Chayen, E. Saridakis, Protein crystallization: from purified protein to diffractionquality crystal, Nat. Methods 5 (2) (2008) 147-153.

[20] A. Kostro, M. Geiger, J.L. Scartezzini, A. Schüler, CFSpro: ray tracing for design and optimization of complex fenestration systems using mixed dimensionality approach, Appl. Opt. 55 (19) (2016 Jul 1) 5127-5134.

[21] Y.S. Muzychka, M.M. Yovanovich, Laminar flow friction and heat transfer in noncircular ducts and channels part I: hydrodynamic problem, in: G.P. Celata, B. Thonon, A. Bontemps, S. Kandlikar (Eds.), Compact Heat Exchangers: A Festschrift on the 60th Birthday of Ramesh K. Shah, Grenoble (France) 2002, pp. 123-130.

[22] C.K. Malek, V. Saile, Applications of LIGA technology to precision manufacturing of high-aspect-ratio micro-components and-systems: a review, Microelectron. J. 35 (2) (2004) 131-143.

[23] J. Gong, F. Meyer, Y. Leterrier, A. Kostro, A. Schüler, Dimensional stability analysis of a UV printed polymer microstructure for a novel glazing system, Energy Procedia 122 (2017) 763-768.

[24] S. Nandi, H.H. Winter, Swelling behavior of partially cross-linked polymers: a ternary system, Macromolecules 38 (10) (2005) 4447-4455.

[25] B.A. Miller-Chou, J.L. Koenig, A review of polymer dissolution, Prog. Polym. Sci. 28 (8) (2003) 1223-1270. 